\title{
La actividad académica del estudiante universitario como proceso ocupacional: comprensión desde la terapia ocupacional
}

Clara Duarte Cuervo*

\section{RESUMEN}

El objetivo de este artículo es sensibilizar a la comunidad profesional acerca de la pertinencia de la intervención de Terapia Ocupacional con estudiantes universitarios. Se enfoca desde la comprensión de la escolaridad del joven como proceso ocupacional, describe la disfuncionalidad en el desempeño de su rol e identifica las características del ambiente universitario y las demandas de la actividad académica que determinan la calidad del desempeño ocupacional en esta edad y contexto particular. Resulta este un campo aparentemente inexplorado, pero de una gran amplitud si se toma en cuenta la cantidad de factores de riesgo a los que está expuesto el adolescente, su trascendencia en la construcción del proyecto de vida y la especial vulnerabilidad del ser humano en esta etapa.

\section{ABSTRACT}

This article pretend to call attention of the Occupational Therapy's professional community about the relevance of occupational therapy exercise with university students. It focus in the understanding of academic activity in young like a occupational process. It describes the dysfunction in role performance and identifies the

* Terapeuta Ocupacional. División de Salud Estudiantil. Universidad Nacional de Colombia. 
characteristics of the university environment and the demands of academic activity that determines the quality of occupational performance in this particular age and context. This field is apparently unexplored, but is very long, over count if have in mind the multiple risk factors that are present in the adolescence, the impact of this time in the construction of live project and the special vulnerability of human being in this age.

Tradicionalmente al hablar de la ocupación humana como objeto de estudio de terapia ocupacional, se tiende a ignorar el rol ocupacional del estudiante universitario y el actuar ocupacional propio del adolescente y el joven. Las teorías, así como la descripción de protocolos y programas de intervención se enfocan en el rol del niño como jugador y escolar, el rol del adulto como trabajador y el del anciano como ser dedicado al disfrute del ocio y el tiempo libre. Los trabajos de terapeutas que abordan la población adolescente, se enfocan principalmente en la rehabilitación o el control de riesgos en poblaciones altamente vulnerables por desenvolverse en condiciones de deprivación ambiental o afectiva, exposición al uso de sustancias psicoactivas, delincuencia juvenil o prostituición ${ }^{1}$. No obstante, el abordaje desde el punto de vista de la ocupación propia del joven, de las demandas inherentes al ejercicio de dicho rol y de las condiciones ambientales y sociales que afectan y determinan su desempeño, es más bien tímida o nula.

La cifra de jóvenes que accede a la educación superior aumenta progresivamente con el paso del tiempo. A pesar del limitante fundamental que representa su alto costo, el proyecto de vida de los jóvenes hoy incluye, en la mayoría de casos, el paso por algún tipo de educación superior antes o de manera simultánea con el ingreso al mundo del trabajo. Para dar un ejemplo, solo en los últimos dos años el número de aspirantes a ingresar a la Universidad Nacional de Colombia ha aumentado alrededor de un $8 \%^{2}$. Las demandas del mercado laboral, así como el

\footnotetext{
${ }^{1}$ En una revisión de los artículos publicados por la Asociación Colombiana de Terapia Ocupacional entre 1996 y 1999 solo se encontraron cuatro artículos relacionados con la intervención en población adolescente y joven. De ellos dos hacen referencia a intervenciones con población con discapacidad, uno a factores de riesgo psicosocial para violencia juvenil y el último a orientación ocupacional en estudiantes de grado décimo. Por otra parte, el artículo "Perspectiva del Ejercicio Profesional del Terapeuta Ocupacional en el Área Educativa" hace alusión al rol profesional en la educación básica únicamente.

${ }^{2}$ La dirección Nacional de Admisiones de la Universidad Nacional de Colombia reporta que la cifra pasó de 111.766 aspirantes en el año 1998 a 121.129 en el año 2000.
} 
deseo de mejorar el nivel de vida, han llevado a que las personas den una mayor importancia al mejoramiento de su cualificación laboral o profesional a través de la formación técnica, tecnológica o profesional. En consecuencia, cada vez son menos las personas que al finalizar sus estudios secundarios no consideran la opción de acceder a la educación superior.

En el pasado el joven se convertía tempranamente en trabajador, actualmente en cambio, la vida como estudiante se prolonga unos años más, sin embargo, el hecho de seguir siendo estudiante no implica que el desempeño del rol se conserve estático.

El ingreso a cualquier sistema de educación superior y específicamente a la universidad, implica cambios drásticos en el estilo de vida que demandan un ajuste o adaptación, la vivencia de un nuevo rol con demandas ocupacionales, ambientales y sociales diferentes y la exposición a factores de riesgo específicos; en consecuencia, lleva a la transformación de comportamientos, hábitos y acciones ocupacionales. De la misma manera, el cambio en las demandas inherentes al rol exige que el joven demuestre habilidades cognoscitivas, físicas, sociales y psicológicas que le permitan no solo adaptarse al nuevo medio, sino paralélamente, mantener un desempeño que le garantice responder a las demandas académicas y posteriormente, desempeñarse eficientemente como trabajador. Esta etapa entonces, no solo es un paso que se añade a la vida del joven contemporáneo, sino se convierte en el pilar sobre el cual este edifica su futuro y que determina las características de las ocupaciones que llenaran su vida en etapas posteriores.

Múltiples factores hacen que el estudiante en la educación superior sea especialmente vulnerable a ver interrumpido o deteriorado el desempeño de su rol, por lo tanto, la intervención del terapeuta ocupacional sobre esta población dista mucho de ser limitada o discreta.

Vista la escolaridad del joven como proceso ocupacional", "conlleva demandas sensoriomotoras, cognoscitivas y socioafectivas", así mismo, se encuentra determinado (y a su vez las influencia) por un lado, por las capacidades, potencialidades y necesidades humanas, y por otro, por las características sociales, temporales y culturales del medio en el cual la actividad académica se desarrolla.

${ }^{3} \mathrm{El}$ concepto de proceso ocupacional obedece a lo propuesto en el Modelo del Desempeño Ocupacional Realizante. 
?niendo en cuenta estos principios, se puede caracterizar y analizar el desempeño zupacional del estudiante universitario, así como explicar y entender los factores se determinan la calidad de las experiencias durante esta etapa.

a disfunción ${ }^{4}$ en el desempeño académico se manifiesta básicamente en un bajo ndimiento, aunque este puede entenderse como representación última que lleva as de si otros niveles de disfunción. Dichos niveles pueden entenderse como conformidad con la elección ocupacional, desmotivación frente a contenidos o :tividades específicas, falta de habilidad sentida frente a acciones académicas o ofesionales concretas, dificultad para la comprensión o aplicación de contenidos, unflictos con docentes (de carácter personal o estrictamente pedagógico), isentismo y deserción escolar, incapacidad para distribuir el tiempo entre las :tividades académicas y extra-académicas, incapacidad para adaptarse e tegrarse al medio universitario, estrés ocupacional y otras enfermedades o :cidentes derivados del ejercicio de la actividad académica.

sniendo en cuenta que la calidad del desempeño ocupacional depende del "grado z armonía entre las características personales, las demandas ocupacionales y su terrelación con el medio ambiente" ${ }^{5}$, los estados o señales de disfunción en el zsempeño enunciados anteriormente, obedecen a los imbalances que entre estos ctores se suceden en el actuar del estudiante universitario. A continuación se ralizará cada uno de ellos.

unque las habilidades requeridas para el desempeño como estudiante son ásicamente de tipo cognoscitivo, en la educación superior las demandas zupacionales varían de manera significativa de acuerdo al programa académico , carrera), la estructuración del currículo, los modelos y métodos pedagógicos

\footnotetext{
je toman aquí como referente los conceptos de Trujillo (1994) quien definió los estados del sempeño ocupacional: "Desempeño ocupacional disfuncional: cuando la persona a través de sus racterísticas personales no responde satisfactoriamente a las demandas que le plantea tanto la upación como el medio ambiente, ocasionado esto por un imbalance entre las variables relacionadas, jor tanto las demandas se constituyen en obstáculos que no permiten que la persona, la ocupación el entorno se beneficie de esta interrelación.

ssempeño ocupacional funcional: Cuando la persona responde satisfactoriamente de conformidad $\mathrm{n}$ los requerimientos de su nivel de desarrollo, las demandas ocupacionales y ambientales de tal anera que en esta interrelación se beneficia de su entorno y también lo enriquece."

'lanteado por Torres (1994) en el artículo: Modelos Operativos para Terapia Ocupacional.
} 
adoptados por instituciones y docentes, la jornada de estudio y el perfil del profesional que se pretende formar.

En cuanto a las características de esta población, se debe partir del hecho que está compuesta básicamente por adolescentes y jóvenes, así por ejemplo, en la Universidad Nacional de Colombia el $46 \%$ de los estudiantres que ingresaron entre 1998 y 2000 tenían entre 15 y 17 años. Esto se asocia en general con buenas condiciones de salud física, pero igualmente indica el paso por una etapa que conlleva una nueva relación con el propio cuerpo, cambios en los vínculos con las figuras de autoridad, mayor identificación con el grupo de pares, búsqueda de independencia económica y psicológica, pero a su vez, negación de la vida adulta. Todo esto finalmente se manifiesta en las actividades en las que se compromete y el interés que pone en una u otra; por una parte el estudiante cuenta con las habilidades que ha desarrollado a lo largo de su vida, especialmente durante la escolaridad previa, así como con las potencialidades que a través de la academia y las elecciones avocacionales serán optimizadas, pero igualmente necesita identificarse y motivarse fuertemente por las ocupaciones para que adquieran un significado coherente con sus intereses inmediatos y su proyecto de vida.

El medio ambiente en el que la actividad académica se desarrolla, posee a su vez características particulares. Se debe partir de que el ambiente universitario varía drásticamente entre ciudades $e$ incluso entre planteles. Los recursos físicos, económicos y humanos de que dispone una universidad, determinan la manera como se vive la academia, el tiempo que se permanece en ella, las actividades curriculares y extracurriculares que allí se realizan, e incluso, los riesgos a los que se ve expuesto el estudiante. Cada universidad posee también características culturales, étnicas y sociales que pueden llegar a influir en la formación de las maneras de actuar y pensar.

Sin embargo, el ambiente académico no se limita al campus o claustro universitario, sino que se extiende a los lugares habituales de estudio en el hogar y fuera de él y aquellos en que el estudiante debe realizar prácticas. Estos igualmente poseen condiciones particulares que limitan ofacilitan el aprovechamiento y generan riesgos específicos. En este sentido, entran a jugar un papel importante las condiciones económicas y familiares, así como las características físicas y de ubicación de la vivienda y otros lugares en que el estudiante se desenvuelve, ya que estas a su vez influyen en la calidad de las experiencias ocupacionales que en ellos se desarrollan. 
No se puede olvidar que en esta edad se dedica buena parte del tiempo a la socialización y la diversión, lo que hace que el uso de los espacios universitarios se diversifique, y si este así lo permite, el estudiante logre una mayor apropiación y disfrute del mismo. Las actividades que el joven selecciona para ocupar su tiempo libre $^{6}$ y la frecuencia con que las realiza, facilitan el desarrollo de ciertas habilidades (físicas, cognoscitivas, artísticas, sociales) y el afianzamiento de destrezas, muchas de las cuales se transfieren a otras ocupaciones o se conservan como pasatiempo por el resto de la vida.

De la misma manera, la participación en ciertas actividades relacionadas con el ocio y la diversión, trae consigo la exposición a riesgos de diferente tipo, entre los que se incluyen el abuso del alcohol y otras sustancias psicoactivas, el contagio de enfermedades de transmisión sexual y los embarazos no planeados, que pueden afectar a corto y largo plazo el estado de salud físico y la estabilidad emocional, o llevar al estudiante a replantear su proyecto de vida o a asumir responsabilidades para las que aún no se siente preparado.

El Cuadro 1 resume la relación entre algunas características del ambiente universitario y demandas de la actividad académica y la respuesta del estudiante frente a las mismas.

Visto de esta manera, en términos de proceso ocupacional, se amplia significativamente el panorama de influencias que sortea un estudiante en el desempeño de su rol. Resulta entonces una tarea inmensa identificarlas en cada medio y caso particular con el fin de dar respuestas efectivas y concretas a las necesidades del estudiante que ve vulnerado o en riesgo su desempeño.

Teniendo en cuenta lo anterior, las acciones profesionales del terapeuta ocupacional en esta área, como parte de un equipo interdisciplinario, van desde la orientación profesional hasta la reorganización del desempeño en el momento del ingreso, el egreso o el reingreso a la academia, pasando por la identificación y el control de riesgos derivados del ejercicio de actividades particulares, la identificación de deficiencias frente a demandas específicas, la orientación para el desarrollo o

${ }^{6}$ Entendidas aquí a la manera de López Andrada (citado por Ponce de León, 1998) que las define como"actividades libres que potencian el desarrollo personal". 


\title{
compensación de las mismas y la integración, reintegración y orientación de estudiantes con discapacidad.
}

\author{
Cuadro 1. Relación entre las demandas del ambiente y la actividad académica \\ y la respuesta del estudiante frente a las mismas.
}

\begin{tabular}{|c|c|c|}
\hline $\begin{array}{l}\text { Características del ambiente universitario } \\
\text { y demandas de la actividad académica }\end{array}$ & & $\begin{array}{c}\text { Respuesta del estudiante frente } \\
\text { a las demandas }\end{array}$ \\
\hline $\begin{array}{l}\text { Horarios más flexibles: } \\
\text { Posibilidad de escoger entre varias opciones para } \\
\text { su organización. }\end{array}$ & $\Rightarrow$ & $\begin{array}{l}\text { Percepción de mayor tiempo libre. } \\
\text { Mayor autonomía para organizar el tiempo. } \\
\text { Mayor permanencia fuera del hogar, lo que lleva en } \\
\text { ocasiones a tener conflictos con los padres. }\end{array}$ \\
\hline $\begin{array}{l}\text { Currículos flexibles que dan la oportunidad de ver } \\
\text { materias fuera del área específica de la carrera y } \\
\text { de profundizar en contenidos de acuerdo a los } \\
\text { gustos e intereses individuales. }\end{array}$ & $\Rightarrow$ & $\begin{array}{l}\text { Capacidad de expresar los gustos e intereses } \\
\text { personales. El estudiante decide los conocimientos } \\
\text { y habilidades que desea adquirir o reforzar } \\
\text { Tendencia a comprometerse con demasiadas } \\
\text { actividades. }\end{array}$ \\
\hline $\begin{array}{l}\text { La responsabilidad frente a la propia educación } \\
\text { recae sobre el estudiante: } \\
\text { Se le asignan deberes en investigación, elaboración } \\
\text { de proyectos y estudio personal. } \\
\text { No hay mayor control en cuanto a horarios } \\
\text { presenciales y no presenciales. } \\
\text { Se le da orientación, pero la calidad de los } \\
\text { resultados depende básicamente de su propio } \\
\text { esfuerzo. } \\
\text { Falta de recursos económicos o de apoyo familiar. }\end{array}$ & $\Leftrightarrow$ & $\begin{array}{l}\text { Acumulación de deberes académicos. } \\
\text { Sensación de que el tiempo no alcanza para } \\
\text { cumplirlos. } \\
\text { Trabajo académico nocturno. } \\
\text { Carga mental elevada. } \\
\text { Desorganización del tiempo. } \\
\text { Alteración de los hábitos de sueño y alimentación } \\
\text { En algunos casos la responsabilidad económica es } \\
\text { asumida total o parcialmente por el estudiante: } \\
\text { Necesidad de dedicar el tiempo libre al trabajo } \\
\text { remunerado. } \\
\text { Estrés y ansiedad. }\end{array}$ \\
\hline $\begin{array}{l}\text { Mayor nivel de exigencia académica y cambios en } \\
\text { los métodos de evaluación }\end{array}$ & $\Rightarrow$ & $\begin{array}{l}\text { Malas bases académicas o identificación con otros } \\
\text { métodos de enseñanza y evaluación. } \\
\text { Sensación de incompetencia. } \\
\text { Estrés y ansiedad. }\end{array}$ \\
\hline $\begin{array}{l}\text { Falta de estrategias de información y control para } \\
\text { el manejo de riesgos derivados del ejercicio de } \\
\text { actividades específicas. } \\
\text { Exposición a riesgos físicos, químicos, mecánicos, } \\
\text { biológicos, ergonómicos y psicosociales. } \\
\text { Exposición a ambientes y situaciones desconocidos } \\
\text { para el estudiante, en los que se les exige un actuar } \\
\text { profesional. }\end{array}$ & $\Leftrightarrow$ & $\begin{array}{l}\text { Baja preparación o estabilidad psicológica para } \\
\text { enfrentar medios y situaciones que pueden resultar } \\
\text { traumáticos. } \\
\text { Adquisición de enfermedades de origen profesional. } \\
\text { Accidentes durante el manejo de equipos y mate- } \\
\text { riales. }\end{array}$ \\
\hline $\begin{array}{l}\text { Pocas oportunidades en el mercado laboral. } \\
\text { Desempleo. } \\
\text { Campos de acción limitados. }\end{array}$ & $\Leftrightarrow$ & $\begin{array}{l}\text { Desconocimiento de las propias habilidades e inte- } \\
\text { reses. } \\
\text { Falta de orientación profesional. } \\
\text { Desconocimiento de las profesiones. } \\
\text { Dudas en cuanto a la elección profesional. } \\
\text { Ansiedad frente al panorama laboral. }\end{array}$ \\
\hline
\end{tabular}




\section{BIBLIOGRAFÍA}

1. Asociación Colombiana de Terapia Ocupacional. Revistas Ocupación Humana. Revisión revistas publicadas entre 1996 y 1999. Bogotá.

2. Universidad Nacional de Colombia. Dirección Nacional de Admisiones. Guía del Aspirante. Bogotá, 2000.

3. Trujillo Rojas, Alicia. "Desempeño Ocupacional Realizante: Un Modelo Sobre los Propósitos de la Terapia Ocupacional". En: Revista Ocupación Humana. Vol. 6 No. 2. Bogotá, 1995.

4. Trujillo Rojas, Alicia. "Modelo 'Desempeño ocupacional y realización humana': su origen y alcances". En: Universidad Nacional de Colombia - Departamento de Terapias. Terapia Ocupacional y Universidad. Bogotá: 1994.

5. Torres de Tovar, Martha L. "Modelos operativos para Terapia Ocupacional". En: Terapia Ocupacional y Universidad. Universidad Nacional de Colombia Departamento de Terapias. Bogotá, 1994.

6. Universidad Nacional de Colombia. División de Salud Estudiantil. Área de Trabajo Social. Cáracterización socioeconómica de ingreso, estudiantes de pregrado. Bogotá, 2001.

7. Ponce de León, Ana. Tiempo libre y rendimiento académico. Ed. Logrono. España, 1998. 